\title{
Editorial
}

\section{Computational Science in Smart Grids and Energy Systems}

\author{
Hongjie Jia, ${ }^{1}$ Ned Djilali, ${ }^{2}$ Xinghuo Yu, ${ }^{3}$ H. D. Chiang, ${ }^{4}$ and Gongnan Xie ${ }^{5}$ \\ ${ }^{1}$ Key Laboratory of Smart Grid of Ministry of Education, School of Electrical Engineering and Automation, \\ Tianjin University, Tianjin 300072, China \\ ${ }^{2}$ Institute for Integrated Energy Systems, University of Victoria, P.O. Box 3055, Victoria, BC, Canada \\ ${ }^{3}$ Platform Technologies Research Institute, RMIT University, City Campus, P.O. Box 2476, Melbourne, Australia \\ ${ }^{4}$ School of Electrical and Computer Engineering, Cornell University, 328 Rhodes Hall, Ithaca, NY 14853-3501, USA \\ ${ }^{5}$ School of Mechanical Engineering, Northwestern Polytechnical University, Xian 710072, China \\ Correspondence should be addressed to Hongjie Jia; hjjia@tju.edu.cn
}

Received 9 October 2014; Accepted 9 October 2014

Copyright (c) 2015 Hongjie Jia et al. This is an open access article distributed under the Creative Commons Attribution License, which permits unrestricted use, distribution, and reproduction in any medium, provided the original work is properly cited.

\section{Introduction}

Computational science for smart grids (SG) and energy systems (EG) pertains to the development of mathematical models, numerical simulations, quantitative analysis, and optimization techniques for large-scale, complex, dynamic, and interconnected systems. As one of the most important integrated energy systems in the future, the "smart grid" allows integration of various renewable energy resources with communication and information technologies to meet spiraling growth of load demands. Smart grids will require new methods to ensure user interaction, measurement and management of massive amounts of data, and cyber security. Rigorous reliability and security operation standards for smart grids will require novel computational science technologies for online modeling, estimation, analysis, control, optimization, decision support, and regulation of different forms of energy generation and storage utilization.

The primary objective of this special issue is to examine and disseminate state-of-the-art research and development in the application of computational science technologies in smart grids and energy systems (SG\&ES) and identify future research directions.

\section{Topics Covered in This Issue}

Topics covered in this issue are (1) SG\&ES modelling and simulation, (2) SG\&ES planning and operation, (3) SG\&ES stability and security, and (4) control design. Details are as follows.

\subsection{SG\&ES Modelling and Simulation}

2.1.1. Modelling of Devices, Components, and System Constraints. X. Zhang et al. provide a new model of ultracapacitors to describe their electrical behaviors; C. Cai et al. propose a novel asynchronous third-order transient machine model with consideration of the frequency characteristic; J. Liu et al. derive a practical approach for power oscillation classification by identifying real-time power oscillation curves; J. Xiao et al. give the model definition of distribution system security region to properly consider security constraints in system planning and operation.

2.1.2. Forecasting Model and Method. C. Zhou and X. Chen present adaptive combination forecasting model of China's energy consumption; W. Li and H. Xie propose a geometrical variable weights buffer $\mathrm{GM}(1,1)$ model to forecast China's energy consumption.

2.1.3. Simulation and Analysis. D. P. Chassin et al. propose an agent-based simulation framework for smart grids and integrated energy systems based on the GridLAB-D platform. J. Zhao et al. present an improved topology-based network model to reflect the structural characteristic of power grid properly. M. A. A. Hamad et al. provide a suitable model to 
carry out the Lie group analysis on Brownian motion and thermophoresis effect on free convective boundary-layer flow on a vertical cylinder embedded in a nanofluid-saturated porous medium.

\subsection{SG\&ES Planning and Operation}

2.2.1. Transmission Network Planning and Operation. $\mathrm{H}$. Zhang et al. present a new method based on multiscale support vector regression, and G. Sun et al. present a new method based on relevance vector machine to improve the precision of wind power/speed forecasting; Y. Hu et al. consider uncertainty and correlation of wind power in their transmission network expansion planning; M. Delfanti et al. study the suitability of extending frequency control to renewable energy sources integration with energy storage systems and consider the requirements for the European energy system; F. Dong et al. provide some survivability index to determine the core backbone grid; J. Yang et al. propose a real-time pricing-based scheduling strategy for smart grid operation based on game theory.

2.2.2. Distribution Network Planning and Operation. H. Liu et al. and S. Ge et al. analyze the impact of DGs on load supply capability and reliability assessment in smart distribution network; Z. Wang et al. study the distribution network security based on $K(N-1+k)$ criterion; S. Wang et al. describe an improved substation location and sizing method based on the weighted Voronoi diagram and the transportation model for urban power networks; H. Jiang et al. present an improved multiobjective genetic algorithm to solve the timeof-use pricing problem; Q. Xu et al. evaluate the potential of electric vehicles (EV) in peak load shifting in future SG\&ES systems; J. Wang et al. give a long-term distribution system maintenance schedule through particle swarm optimization and tabu search method.

\subsubsection{Renewable Energy Resource Integration and Microgrid.} $Z$. Cheng et al. propose a novel method based on finite difference extended Kalman filter to accurately estimate the state of charge (SOC) of the battery in a smart grid; W. Fan et al. provide a microgrid design scheme to achieve low-voltage ride-through capability; Q. Tang et al. give a multiobjective optimal operation scheme for the island microgrid with wind, photovoltaic (PV), diesel, storage, and desalination; B. Li et al. derive a novel frequency calculation algorithm based on extended Kalman filter to track dynamic frequency and islanding detection of the microgrid; W. Liu et al. present a comprehensive environmental-economic dispatch method for microgrid to minimize its generation summation and emission costs.

2.3. SG\&ES Stability and Security. H. Ren et al. give a probabilistic method to evaluate the influence of distributed generation (DG) on system voltage stability and energy losses; Q. He et al. provide a de-icing method for overhead transmission line to improve the safety and reliability of power grids; $\mathrm{X}$. Yu et al. derive a method to determine oscillation emergence bifurcation in SG\&ES system; L. Wang proposes an improved anonymous remote authentication scheme to reinforce the password authentication and update scheme in smart grids. A novel method based on timescale decomposition and singular perturbation theory is presented by W. Zhang et al. to perform the stability analysis of flywheel energy storage systems.

2.4. SGeES Control Designing. D.-H. Li et al. present a new control scheme for photovoltaic grid power generation system to reduce the harmonic output; $\mathrm{T}$. Cao et al. propose a novel bridgeless bipolar-gain pseudo-boost control scheme for PFC converter to achieve higher efficiency; X. Chen and Y. Li present a nondestructive islanding detection method for microgrid to avoid serious transient process during DG's control strategy transformation; T. Bei et al. design a novel high-gain interleaved boost DC/DC converter with coupled inductor and switched capacitor; F. Dong et al. design an optimal subsynchronous damping controller based on improved biogeography-based optimization algorithm to enhance the dynamic performance of power system; Y. Che et al. propose a two-voltage stage topology with boost converter to improve the efficiency of small wind grid inverters. A new fault diagnosis method is proposed for PV arrays by Z . Cheng et al. to minimize the number of sensors number; protection principles and schemes for DC distribution system with DGs are thoroughly investigated and verified by S. Xue et al. in their studies.

\section{Acknowledgments}

We would like to thank all the authors who have contributed to this special issue and the reviewers involved in the refereeing of the submissions.

Hongjie Jia Ned Djilali Xinghuo Yu H. D. Chiang Gongnan Xie 


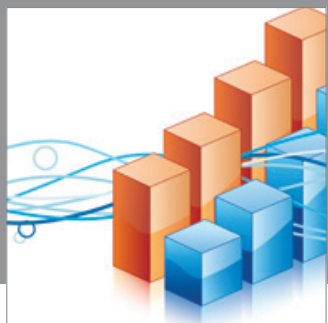

Advances in

Operations Research

mansans

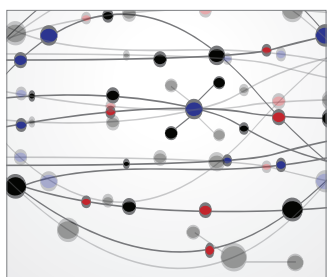

The Scientific World Journal
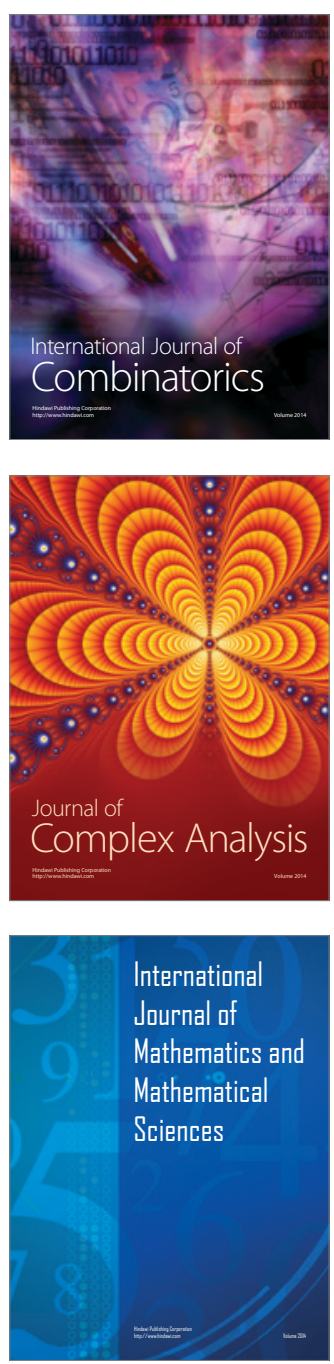
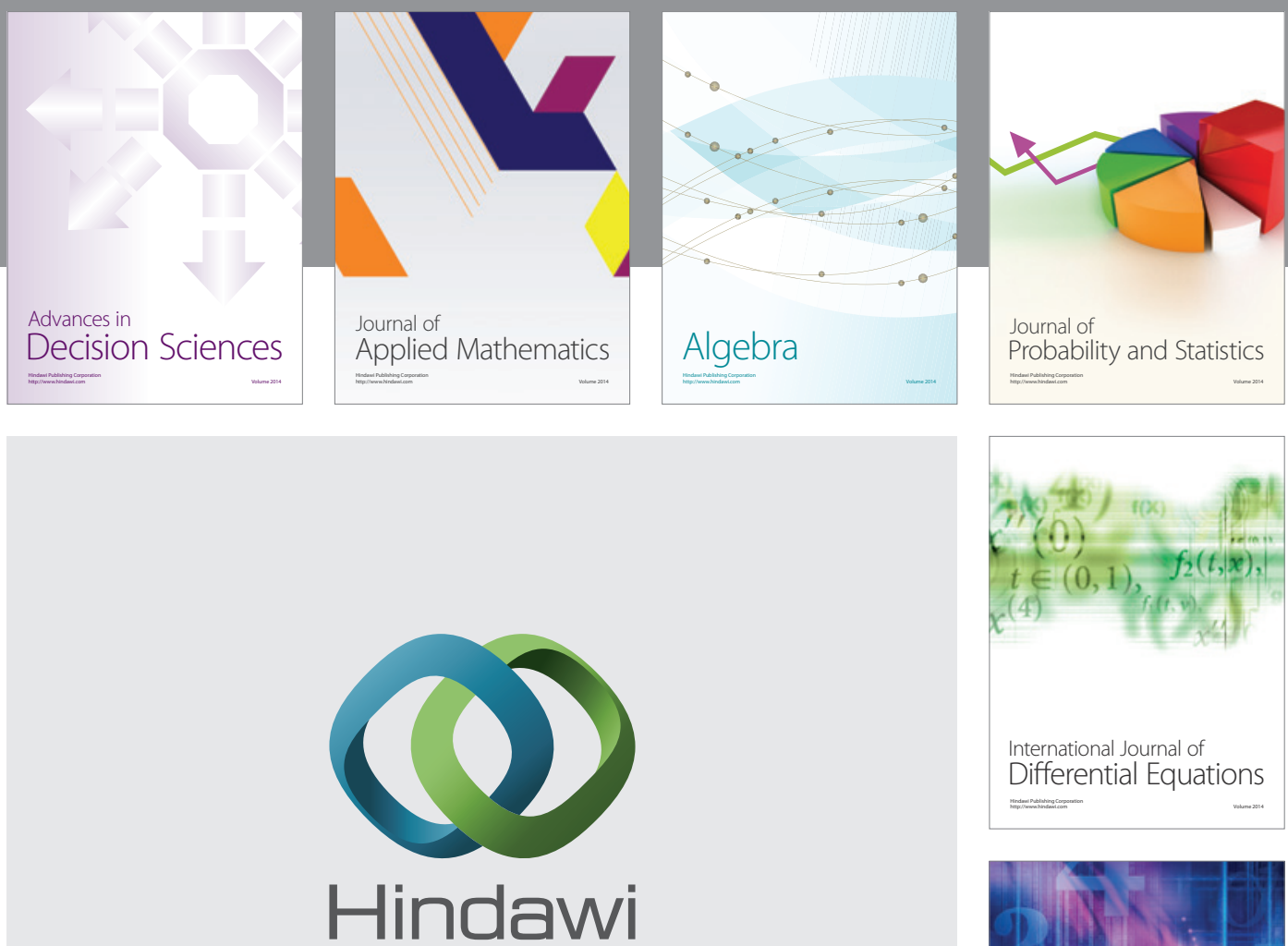

Submit your manuscripts at http://www.hindawi.com
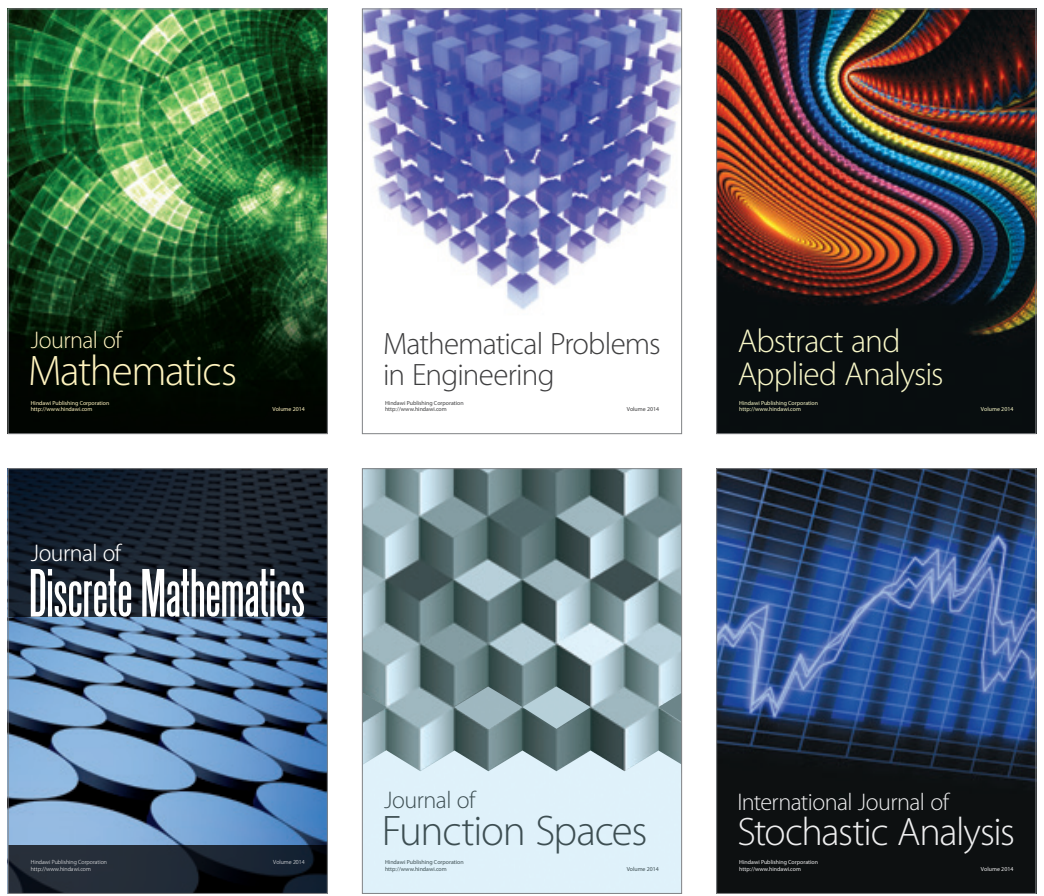

Journal of

Function Spaces

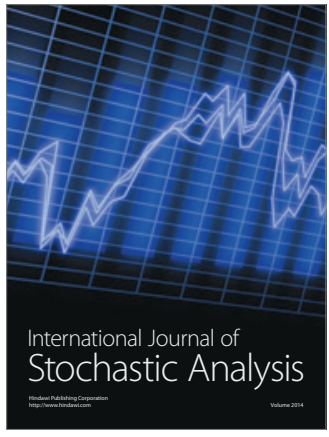

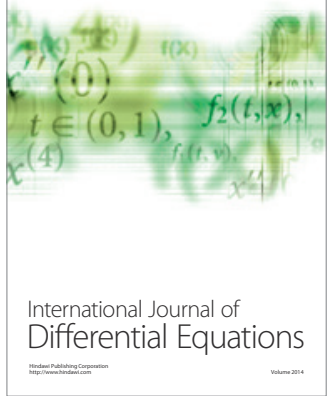
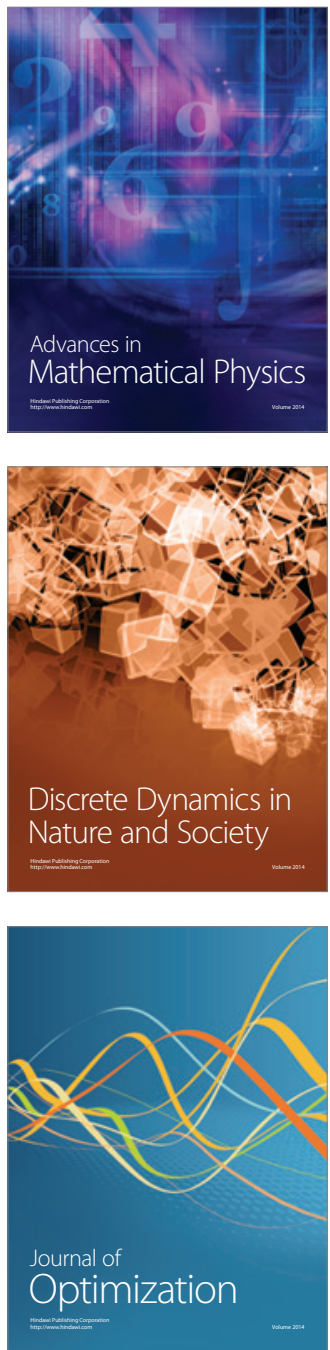